\title{
Silent strokes after thoracoscopic epicardial ablation and catheter ablation for atrial fibrillation: not all lesions are permanent on follow-up magnetic resonance imaging
}

\author{
Hana Malikova ${ }^{1,2 \#} \wedge$, Karin Kremenova ${ }^{1 \#}$, Petr Budera $^{3} \wedge$, Dalibor Herman $^{4} \wedge$, Jiri Weichet $^{1 \wedge}$, \\ Jiri Lukavsky ${ }^{5}$, Pavel Osmancik ${ }^{4} \wedge$
}

${ }^{1}$ Department of Radiology, Faculty Hospital Kralovske Vinohrady and Third Faculty of Medicine, Charles University, Prague, Czech Republic; ${ }^{2}$ Department of Anatomy, Second Faculty of Medicine, Charles University, Prague, Czech Republic; ${ }^{3}$ Department of Cardiac Surgery, Faculty Hospital Kralovske Vinohrady and Third Faculty of Medicine, Charles University, Prague, Czech Republic; ${ }^{4}$ Department of Cardiology, Faculty Hospital Kralovske Vinohrady and Third Faculty of Medicine, Charles University, Prague, Czech Republic; ${ }^{5}$ Faculty of Arts, Charles University, Celetna 20, 11000, Prague, Czech Republic

\#These authors contributed equally to this work.

Correspondence to: Hana Malikova. Department of Radiology, Third Faculty of Medicine, Charles University in Prague, Faculty Hospital Kralovske Vinohrady, Srobarova 1150/50, 11000 Prague, Czech Republic. Email: hana.malikova@fnkv.cz.

Background: Invasive treatments for atrial fibrillation (AF) pose a risk of ischemic stroke due to periprocedural brain embolization, which may be manifest or silent. The primary aim of our study was to compare the rate of silent strokes after percutaneous catheter-based and thoracoscopic epicardial ablation for AF. The secondary aim was to evaluate the development of silent strokes over time.

Methods: We included 39 subjects (aged 64.1 \pm 8.9 years) treated for persistent symptomatic AF with thoracoscopic ablation and 30 subjects (aged $64.1 \pm 10.5$ years) treated for paroxysmal or persistent symptomatic AF with catheter ablation. Subjects underwent brain MRI before and early after the ablation, moreover, the surgical group underwent late MRI 6 months after therapy. On early MRI, the presence of silent strokes and their number and size were evaluated. On late MRI, transformation of previously-detected acute ischemic lesions into chronic infarction or their reversibility were assessed.

Results: Initially, different chronic ischemic findings were found in $64 \%$ of patients from the surgical group and in 70\% from catheter group. Early MRI results: acute ischemic lesions were detected in 2 (6.7\%) subjects (overall 3 lesions sized $<5 \mathrm{~mm}$ ) in the catheter group and in 17 (43.6\%) subjects in surgical group. Most subjects in the surgical group showed multiple lesions (88\%); 195 lesions were detected, a median 6 (IQR 8) lesions per case. Eighty-two percent of lesions were $<5 \mathrm{~mm}, 12 \% 5-10 \mathrm{~mm}, 5 \% 10-30 \mathrm{~mm}$, and $2 \%$ were large territorial ischemia. Only 1 case was symptomatic, the rest were silent strokes. On late MRI, $53.5 \%$ of all acute lesions were reversible. Lesions $<5 \mathrm{~mm}$ were reversible in $63.1 \%$ of cases, lesions $5-10 \mathrm{~mm}$ were reversible in $21.7 \%$ and all lesions larger than $10 \mathrm{~mm}$ persisted. In $29.4 \%$ of patients all acute ischemic lesions were fully reversible.

Conclusions: Periprocedural silent strokes were significantly more common after thoracoscopic epicardial ablation compared to catheter ablation considering both the number of affected patients and number of lesions. The majority of acute ischemic brain lesions were small, up to $5 \mathrm{~mm}$ in diameter, roughly half of which were reversible. Reversibility of acute ischemic lesions decreased with size. However, in $29.4 \%$ of affected patients, all lesions were fully reversible.

\footnotetext{
^ORCID: Hana Malikova, 0000-0002-5453-1347; Karin Kremenova, 0000-0001-5546-1136; Petr Budera, 0000-0001-5547-8704; Dalibor Herman, 0000-0002-7436-1154; Jiri Weichet, 0000-0001-7321-8850; Jiri Lukavsky, 0000-0002-1082-229X; Pavel Osmancik, 0000-0003$0482-4448$.
} 
Keywords: Acute ischemia; lesion; infarction; reversibility

Submitted Jan 08, 2021. Accepted for publication Mar 03, 2021.

doi: $10.21037 /$ qims-21-35

View this article at: http://dx.doi.org/10.21037/qims-21-35

\section{Introduction}

Stroke remains a major health concern and a large public health burden. In the past two decades, the agestandardized rate of stroke mortality has decreased; however, the absolute number of strokes and people who live with its consequences has been increasing every year (1). The proportion of ischemic and hemorrhagic strokes is about $91 \%$ to $9 \%$, respectively, in high income countries (1). With regard to ischemic strokes, several classification systems have been proposed. The widely and commonly used TOAST classification divides ischemic stroke into 5 groups: large artery thrombotic strokes, small penetrating artery thrombotic strokes (lacunar strokes), cardiogenic embolic strokes, cryptogenic strokes of unknown cause and strokes associated with other causes (2). There is emerging evidence that the most ischemic strokes are of embolic origin (3), and a cardiac source of embolism has been estimated in about $20 \%$ of ischemic strokes (3). Small brain embolization may be asymptomatic and is termed silent ischemic stroke. However, silent ischemic stroke may not be entirely asymptomatic, as there may be evidence of cognitive, gait or other functional impairments which may not be evident on common bed-side examination (4). Thus, a silent stroke may be a cerebral infarction that was unnoticed, overlooked or disregarded (4).

It is well known atrial fibrillation (AF) poses a risk of ischemic stroke, and stroke contributes significantly to the increased mortality associated with $\operatorname{AF}(5,6)$. Apart from manifest strokes, silent strokes are also found in patients that suffer from AF. The treatment of AF consists of antiarrhythmic drug therapy or ablation procedures, mainly catheter-based pulmonary venous isolation. Catheter-based pulmonary venous isolation has been shown to be superior to treatment by antiarrhythmic drugs in randomized studies (7). However, the efficacy of catheter ablation in patients with the non-paroxysmal form of AF remains limited. Based on the efficacy of the surgical maze procedure, that consists of exactly described set of lesions in atria being performed during open chest surgery on cardiopulmonary bypass, its less invasive variation, i.e., thoracoscopic epicardial ablation have been introduced (8). As in any invasive cardiac procedure, ablation poses a potential risk for periprocedural embolization, especially to the brain (9). Complication rates including brain embolization may differ significantly between different invasive ablation procedures. A comparison of silent stroke risk between percutaneous catheter-based and thoracoscopic epicardial ablation has never been performed. Therefore, our study primarily aimed to assess the rate of silent strokes between these two different ablation strategies. The secondary aim was to evaluate the development of potential new ischemic lesions by magnetic resonance imaging (MRI) 6 months after treatment.

\section{Methods}

\section{Patient selection}

We prospectively included all consecutive patients treated with thoracoscopic epicardial ablation for persistent or longstanding persistent symptomatic AF at our institution from March 2016 to March 2019. All patients were scheduled for preoperative MRI 24 hours before surgery, early followup MRI 24 hours after surgery and for late follow-up MRI 6 months after surgery.

The catheter ablation group was selected from patients who underwent catheter ablation for $\mathrm{AF}$ at our institution from November 2017 to May 2018, and were matched by the age and sex to the surgically-treated group. These patients suffered from symptomatic paroxysmal or persistent $\mathrm{AF}$; the latter refused the thoracoscopic procedure. All subjects in this group underwent preprocedural and early postprocedural MRI. This group of patients was not scheduled for late MRI follow-up, due to the results of the early follow-up MRI (see below).

Common inclusion criteria for interventional therapy (both thoracoscopic epicardial and catheter ablation) of AF were as follows: age $>18$ years, symptomatic AF (paroxysmal, persistent, or long-standing persistent) refractory to at least one antiarrhythmic medication, an 
absence of significant structural heart disease (coronary or valve disease). For exclusion of significant structural heart disease, transthoracic echocardiography was performed in all patients; transesophageal echocardiography was performed only in subjects with suboptimal transthoracic echocardiography visualization, or suspicion of valve disease. Coronary angiography, or computed tomography coronary angiography was done in all surgical candidates, in patients referred to catheter ablation only in a presence of symptoms suspicious for coronary artery disease. Patients in both groups who refused or were unable to undergo the MRI exam were excluded.

In all patients, relevant medical histories as well as relevant data related to periprocedural events were collected.

The study was approved by the Ethics committee of Faculty Hospital Kralovske Vinohrady, Prague, Czech Republic. Signed, informed consent was provided by all patients that participated in the study.

\section{Thoracoscopic epicardial ablation technique}

The technical details of the procedure have been published previously by Budera et al. (8). Surgical ablations were performed using a thoracoscopic, right-sided, offpump, epicardial approach. The goal was to create a circumferential lesion anterior to the pulmonary vein to isolate all of the pulmonary veins together with the posterior aspect of the left atria (i.e., a "box-lesion" set). This continuous lesion was created using a versapolar (unipolar/bipolar), linear radiofrequency COBRA Fusion 150 ablation catheter (Estech, an AtriCure Company, San Ramon, CA, USA). Three or four ablation cycles were performed in both modes (i.e., bipolar and unipolar) with a temperature-controlled energy application setting of $70{ }^{\circ} \mathrm{C}$ and a duration of 60 seconds per cycle. The ablation, performed through the right chest wall, was then followed by a left-sided thoracoscopic occlusion of the left atrial appendage using the AtriClip Pro device (AtriCure Inc., Cincinnati, OH, USA). All oral anticoagulation, novel oral anticoagulants (NOAC) and Warfarin, were stopped 2-5 days before surgery and switched to low molecular weight heparin (LMWH) in prophylactic doses (i.e., nadroparine $0.5 \mathrm{mg} / \mathrm{kg}$ twice daily). Intravenous (I.V.) heparin $(80 \mathrm{IU} / \mathrm{kg})$ was given to all patients during the surgical procedure after dissection, but before starting the ablation. The first application of radiofrequency energy was performed after the verification of activated clotting time (ACT) $>300$ seconds. The ACT values were measured repeatedly every 20 minutes and additional heparin boluses were given if ACT fell below 300 seconds. After surgery, in the intensive care unit, a continuous infusion of I.V. heparin was given with a target of 200 seconds until the next morning, when the first dose of LMWH was given $(0.75 \mathrm{mg} / \mathrm{kg}$ twice daily). Oral anticoagulation was resumed on the second postoperative day (i.e., $48 \mathrm{~h}$ after surgery), if no hemorrhagic complications were present. In patients on Warfarin, additional LMWH was administered until reaching an international normalized ratio (INR) $>2$.

Mean time duration of the thoracoscopic procedure was $170.8 \pm 33.5$ minutes (median 165 minutes). Spontaneous version to sinus rhythms occurred in 11 patients during thoracoscopic procedure, electrical cardioversion was done in 27 patients during or after the surgical procedure $(1.6 \pm 0.7$ version/patient).

\section{Pulmonary venous catheter ablation technique}

All catheter ablations were performed using a threedimensional (3D) mapping system under mild-sedation (fentanyl, midazolam). The left femoral vein was used for the introduction of an $11 \mathrm{~F}$ sheath for the intracardiac echocardiography (ICE) catheter and a $7 \mathrm{~F}$ sheath for the decapolar coronary sinus catheter. The right femoral vein was used for the introduction of two $8.5 \mathrm{~F}$ transeptal sheaths (SL-1, SJM, St. Paul, MN, USA). Five-thousand IU of heparin was administered before the transseptal puncture, then transseptal puncture was performed using ICE navigation and a further heparin bolus was given to achieve an ACT of $>300$ seconds. ACT was repeatedly checked every 20 minutes, and additional boluses of heparin were given to maintain ACT $>300$ seconds. One of the SL1 transeptal sheaths was used for the insertion of a circular mapping catheter (Lasso, Biosense-Webster Inc., Diamond Bar, CA, USA). This catheter was positioned in both the right and left pulmonary veins, the aim was to demonstrate and confirm the presence of both entrance and exit block at the end of ablation. The second sheath was used for the insertion of a $3.5 \mathrm{~mm}$ irrigated-tip CARTO ablation catheter (ThermoCool Smart Touch, Biosense Webster, USA). Three-dimensional reconstruction of the left atrium was performed, and the ostia of all 4 pulmonary veins were marked. Radiofrequency energy was applied using a ThermoCool Smart Touch catheter and software module that enables contact force sensing with a temperature limitation of $43{ }^{\circ} \mathrm{C}$, radiofrequency energy up to $45 \mathrm{~W}$, and contact force $>5 \mathrm{~g}$. Ablation endpoints were 
pulmonary veins isolation in all patients. In selected nonparoxysmal patients, additional lines (roof, mitral isthmus, cavo-tricuspid isthmus) were also allowed, based on the discretion of treating the electrophysiologist. Regarding the anticoagulation protocol, in patients on NOAC, the last dose of NOAC was administered one day before the procedure (in the morning for rivaroxaban, in the evening for apixaban or dabigatran). All sheaths were removed at ACT $<170$ seconds on the day of the procedure, and a continuous heparin infusion was given with a target activated partial thromboplastin time (APTT) of 50 seconds up to the next morning, when NOACs were readministered. In patients on Warfarin before the procedure, catheter ablation was performed on uninterrupted Warfarin, but only when INR was between 2 and 3 (measured one day before the procedure). Similar to NOAC patients, sheaths were removed at ACT $<170$ seconds; however, no further I.V. heparin was given after the procedure due to effective INR. Warfarin was readministered in the morning after the procedure. Cardioversion was performed in only 2 patients during the procedure in this cohort.

\section{MRI examination of the brain}

All MRI examinations were performed on the same diagnostic 1.5 T scanner (Signa HDx 1.5 T, GE Healthcare, Milwaukee, USA), using an HD 8 Channel High Resolution Brain Array Coil. The following MRI protocols were used: A standard protocol consisting of axial T2-weighted turbo spin-echo (TSE T2 WI; slice thickness $4 \mathrm{~mm}$ ), axial T2weighted fluid attenuated inversion recovery (T2 FLAIR; slice thickness $4.5 \mathrm{~mm}$ ), sagittal T1-weighted spin-echo (SE T1 WI; slice thickness $4 \mathrm{~mm}$ ), axial T2* gradient-echo (GRE T2*; slice thickness $5 \mathrm{~mm}$ ), axial diffusion-weighted imaging (DWI) $[\mathrm{b}=0 / 1,000$; slice thickness $5 \mathrm{~mm}$, apparent diffusion coefficient (ADC) map] sequences for the preoperative MRI and late follow-up MRI. An abbreviated MRI protocol consisting of axial T2 FLAIR (slice thickness $4.5 \mathrm{~mm})$, axial DWI $(\mathrm{b}=0 / 1,000$, slice thickness $5 \mathrm{~mm}$, ADC map) sequences was used for the early follow-up MRI 24 hours after procedures.

\section{Brain MRI evaluations}

The following features were assessed on pretreatment brain MRI:

* The presence of acute/subacute ischemia; * Signs of chronic territorial infarctions;
* Signs of chronic lacunar infarctions (lacuna was defined as cerebrospinal fluid-filled cavities $<15 \mathrm{~mm}$ in diameter surrounded by gliotic tissue);

* Fazekas scale, which was used to semi-quantify nonspecific, T2-hyperintense white matter lesions usually attributed to chronic ischemic disease (10);

* Other significant brain pathology.

On the early posttreatment MRI, the presence of hyperacute/acute ischemic lesions was evaluated; moreover, all acute ischemic lesions were classified according to their size $(<5,5-10,10-30,>30 \mathrm{~mm}$ and large territorial ischemia). Diagnostic MRI criteria for acute ischemia were as follows: a hyperintense lesion on DWI $(b=1,000)$ corresponding with hypointense lesion on $\mathrm{ADC}$ map \pm hyperintense lesion on $\mathrm{T} 2$ FLAIR. All acute ischemic lesions were measured on DWI (axial scans) in their longest diameter.

On the late MRI follow-up 6 months after therapy, the following were evaluated:

* Development of brain ischemia previously detected on the early posttreatment MRI;

* New acute ischemic lesions;

* Progression of chronic ischemic lesions;

* Any other significant brain pathology.

The development of previously-detected acute ischemic brain lesions was assessed into 3 categories:

* Common transformation of acute ischemic lesions into chronic infractions: all evaluated lesions underwent transformation into chronic gliotic T2 FLAIR hyperintense lesions or lacunar/pseudocystic lesions surrounded by gliosis.

* Complete reversibility of all acute ischemic lesions was defined as the absence of any T2 signal abnormalities at the site of the previously (on early MRI) detected lesions.

* Partial reversibility was assessed when some lesions were transformed into chronic lesions on the late MRI, while some resolved completely.

All MRI exams were evaluated by two radiologists (experienced 25 years and 2 years in MRI reading).

\section{Statistics}

Data were expressed as mean $\pm \mathrm{SD}$, median and interquartile range (IQR). We compared the comorbidities and demographic data of both groups using the ChiSquare test or Fisher Exact test for categorical data and a $t$-test for continuous data. Linear or logistic regression was used for testing the predictive value of comorbidities 
Table 1 Patient demographic data

\begin{tabular}{|c|c|c|c|}
\hline Variable & Thoracoscopic epicardial ablation & Catheter ablation & ${ }^{\mathrm{t}} \mathrm{P}$ value \\
\hline Male/female & $22 / 17$ & $17 / 13$ & 0.999 \\
\hline Age (mean \pm SD years) & $64.1 \pm 8.93$ & $64.1 \pm 10.5$ & 0.998 \\
\hline Type of AF persistent/paroxysmal & $39 / 0$ & $11 / 19$ & $<0.001$ \\
\hline Hypertension & 27 & 19 & 0.797 \\
\hline Chronic heart failure & 15 & 4 & 0.041 \\
\hline Coronary artery disease & 4 & 6 & 0.312 \\
\hline History of acute ischemic stroke & 0 & 3 & 0.078 \\
\hline
\end{tabular}

${ }^{\mathrm{I}} \mathrm{P}$ values correspond to t-test (for age) or the Chi-Square test or Fisher Exact tests. AF, atrial fibrillation; No, number; SD, standard deviation.

and demographic data on the presence and/or number of ischemic lesions. $\mathrm{P}$ values $<0.05$ were considered significant. Analyses were performed using R Core Team 2020 (11).

\section{Results}

\section{Patient selection}

In the surgical group treated with thoracoscopic epicardial ablation, 39 patients were included: 22 males (mean age $61.7 \pm 11$ years, median 65.5 years) and 17 females (mean age $67.6 \pm 10$ years, median 70.6 years). All subjects were treated for non-paroxysmal symptomatic AF (23 for persistent and 16 for long-standing persistent).

In the catheter ablation group, 30 subjects were included: 17 males (mean age $61.5 \pm 10$ years, median 63.6 years) and 13 females (mean age $67.6 \pm 10$ years, median 70.6 years). Nineteen subjects suffered from paroxysmal and 11 from persistent AF; no patient had a history of long-standing persistent $\mathrm{AF}$.

The distribution of AF type differed significantly between both groups; see Table 1, which summarizes the selection data, comorbidities and pretreatment characteristics of both groups. The type of AF was not the only significant difference between both groups; surgicallytreated patients more often had a history of heart failure.

\section{MRI data}

\section{Pretreatment MRI data}

No significant differences between both groups were found in the evaluation of chronic ischemic changes on pretreatment MRI. The MRI findings of both groups are provided in more detail in Table 2.

\section{Patients treated with thoracoscopic epicardial ablation}

In $36 \%$ of subjects, the preoperative brain MRI was assessed as completely normal. Multiple non-specific white-matter lesions assessed as Fazekas 1 or 2 were found in $55 \%$ of cases, and in $1(2.5 \%)$ patient multiple chronic territorial infarctions were present. In $5 \%$ of cases, acute ischemic lesions were found ( 3 lesions in 2 patients, up to $10 \mathrm{~mm}$ in diameter). Incidentally, the following significant and possibly clinically-relevant lesions were found: one case of gray matter heterotopia, one case of cortical dysplasia, one cavernoma, one patient suffered from a post-contusion lesions and 2 patients suffered from pronounced brain atrophy (in one case normotensive hydrocephalus was also suspected).

\section{Patients treated with catheter ablation}

In $30 \%$ of subjects, the brain MRI was assessed as completely normal. Multiple non-specific white-matter lesions assessed as Fazekas 1 or 2 were found in $63.3 \%$ of cases and in $1(3.3 \%)$ patient chronic territorial infarctions were present. In one case (3.3\%), subacute solitary ischemic lesions were found (up to $10 \mathrm{~mm}$ in diameter). Incidentally, the following significant and possibly clinically-relevant lesions were found: one case of meningioma and one case of chronic subdural hematoma.

The presence of pretreatment chronic ischemic lesions in both groups was more likely in older patients (logistic regression, OR 1.086, 95\% CI: 1.02-1.15, $\mathrm{P}=0.009$ ). 
Table 2 Pretreatment MRI findings

\begin{tabular}{|c|c|c|c|}
\hline MRI findings & Thoracoscopic epicardial ablation (39 subjects) & Catheter ablation (30 subjects) & ${ }^{\mathrm{I}} \mathrm{P}$ value \\
\hline Fazekas 1 & 13 & 15 & 0.250 \\
\hline Fazekas 2 & 9 & 4 & 0.365 \\
\hline Fazekas 3 & 0 & 0 & $\mathrm{~N} / \mathrm{A}$ \\
\hline Lacunar infarcts & 2 & 1 & $\mathrm{~N} / \mathrm{A}$ \\
\hline Acute ischemic lesions & 2 & 1 & $\mathrm{~N} / \mathrm{A}$ \\
\hline
\end{tabular}

${ }^{\mathrm{I}} \mathrm{P}$ values correspond to Chi-Square test or Fisher Exact tests. MRI, magnetic resonance imaging.

Group A: Patients treated by the thoracoscopic epicardial ablation (number of patients 39)

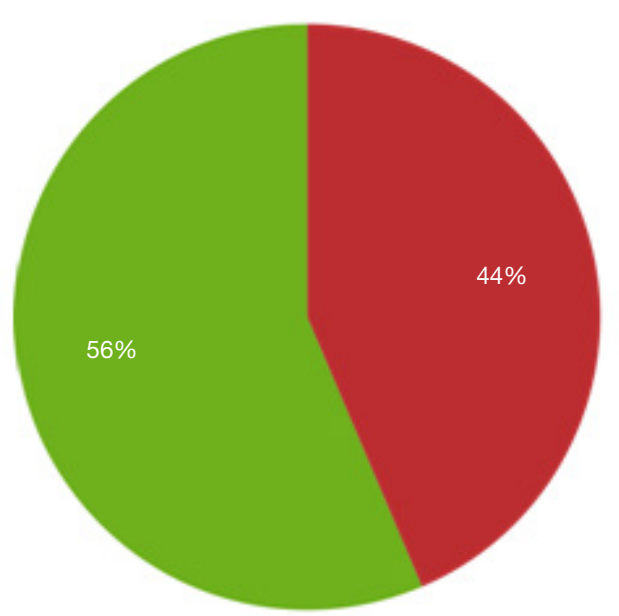

Group B: Patients treated by the catheter pulmonary veins ablation (number of patients 30)

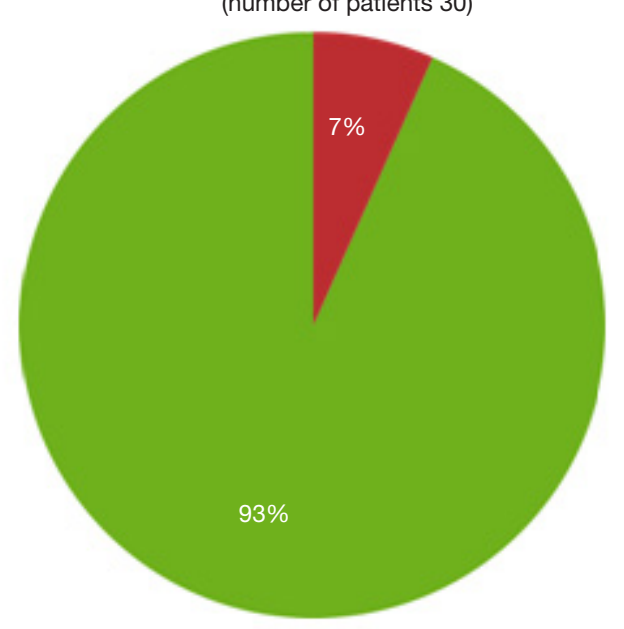

Patients with acute ischemic lesions

Patients without acute ischemic lesions

Figure 1 Early follow-up MRI: occurrence of new ischemic lesions. Graphs demonstrate the much more common occurrence of periprocedural silent strokes in patients treated with thoracoscopic ablation compared with patients after catheter ablation. MRI, magnetic resonance imaging.

\section{Early MRI follow-up evaluation}

There were significantly more acute ischemic lesions after thoracoscopic epicardial ablation compared to catheter ablation. This finding was present if evaluated as the number of affected patients $(\mathrm{P}=0.001)$, or the number of new lesions $(\mathrm{P}<0.001)$, see also Figure 1.

\section{Patients treated with thoracoscopic epicardial ablation}

On early follow-up MRI 195 acute ischemic lesions were detected in 17 (43.6\%) subjects; mean number of lesions per one treated patient was $1.9 \pm 2.7$, the mean number of ischemic lesions per one affected subject was $10 \pm 12$ (median 6, IQR 8). The majority (160 of 195 lesions) of new ischemic lesions were very small $(<5 \mathrm{~mm}) ; 23$ lesions measured $5-10 \mathrm{~mm}$ in diameter, 9 lesions were $10-30 \mathrm{~mm}$ in diameter, and 3 lesions were large territorial ischemia $>30 \mathrm{~mm}$ in diameter. Just one subject suffered from clinically-manifest stroke, the rest were assessed as silent strokes. Acute ischemic lesions were situated in the cerebral hemispheres in all subjects, and in 5 cases small lesions 


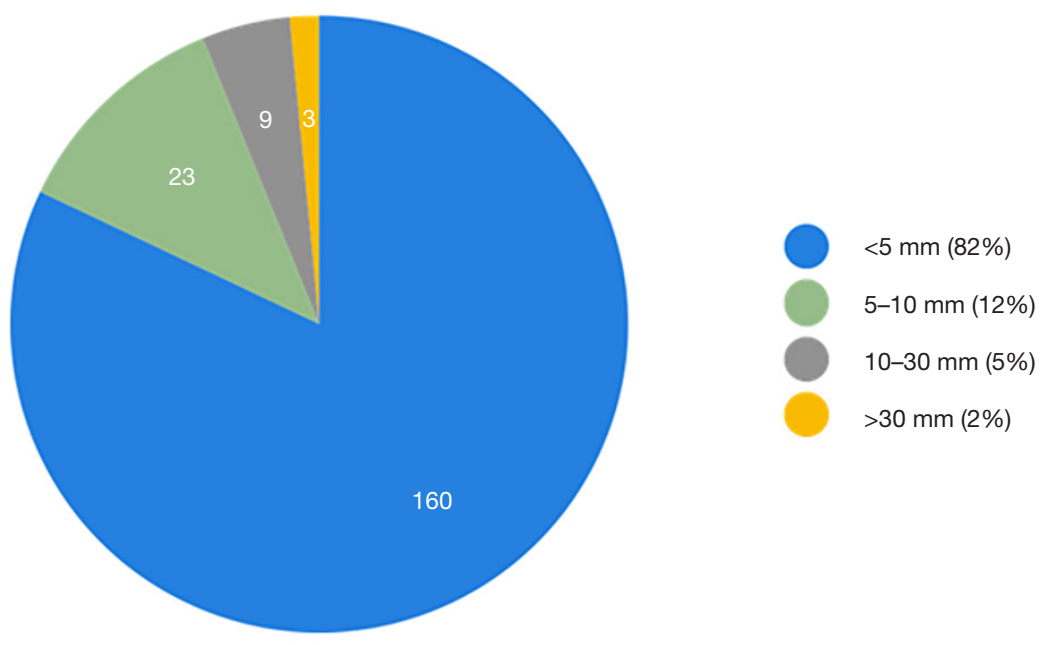

Figure 2 Early follow-up MRI in patients treated with thoracoscopic epicardial ablation. The distribution of lesions according to the size is depicted. The majority of acute ischemic lesions in this group of patients was small ( $<5 \mathrm{~mm})$. Larger lesions $>30 \mathrm{~mm}$ were infrequent. MRI, magnetic resonance imaging.

were also diagnosed in the cerebellar hemispheres. Isolated infratentorial ischemic lesions were not detected. For more details, see Figure 1 and Figure 2. In Figure 3, we demonstrate the findings of multiple small silent strokes of embolic origin. Figure 4 demonstrates MRI findings in the subject with manifest stroke, the patient suffered from hemiparesis and aphasia.

In 6 of 14 patients with normal preoperative brain MRI, acute ischemic lesions were found; in one subject a solitary ischemic lesion was present, in 5 subjects acute ischemic lesions were multiple (5-10 lesions), all lesions were up to $10 \mathrm{~mm}$ in diameter.

A regression analysis was performed between the number of patients with lesions, or number of lesions, as dependent variable, and important baseline and procedural characteristics (such as age, sex, AF type, length of the procedure). No parameters independently and significantly associated with the postprocedural findings of acute lesions were found.

\section{Patients treated with catheter ablation}

On the early follow-up MRI, acute ischemic lesions were detected only in 2 (6.7\%) subjects; overall 3 lesions were found (mean number of lesions per one treated patient was $0.1 \pm 0.4$; the mean number of ischemic lesions per one affected subject was $1.5 \pm 0.7$, median 1.5 ). All lesions were $<5 \mathrm{~mm}$ in diameter; one lesion was situated in the parietal lobe and 2 in the cerebellum.

\section{Late MRI follow-up evaluation}

Only subjects who underwent thoracoscopic epicardial ablation (surgical group) were scheduled for the late follow-up MRI. In 5 (29.4\%) of 17 patients in whom acute ischemic lesions were found on the early follow-up MRI, all lesions fully regressed, thus we did not detect any residual signal changes, gliosis, or pseudocystic lesions at the site of previous acute lesions (on early MRI 18 lesions in these patients were present; 16 lesions were $<5 \mathrm{~mm}$ in diameter and 2 lesions measured $5-10 \mathrm{~mm}$ in diameter). In $9(52.9 \%)$ of 17 patients some residual lesions were apparent on the late follow-up MRI, and were assessed as partially reversible. In the last 3 subjects (7.7\%), chronic transformation of all previously detected acute ischemic lesions into gliotic or pseudocystic lesions was found on the late follow-up MRI; 2 of these patients suffered from chronic territorial infarctions (see also Figure 4). For more details see also Figure 5. More than half (53.5\%) of all acute ischemic lesions were reversible; most were small lesions $<5 \mathrm{~mm}$ in diameter, which were reversible in $63.1 \%$ of cases (see also Table 3, Figures 6,7). Moreover, in one patient, a new acute ischemic lesion was found on the late followup MRI, and in 2 patients, progression of chronic lesions was detected (in all of them the treatment for AF was not successful).

Subjects treated with catheter ablation were not scheduled for the late follow-up MRI as only solitary acute ischemic lesions ( 3 small lesions in 2 patients) were detected 


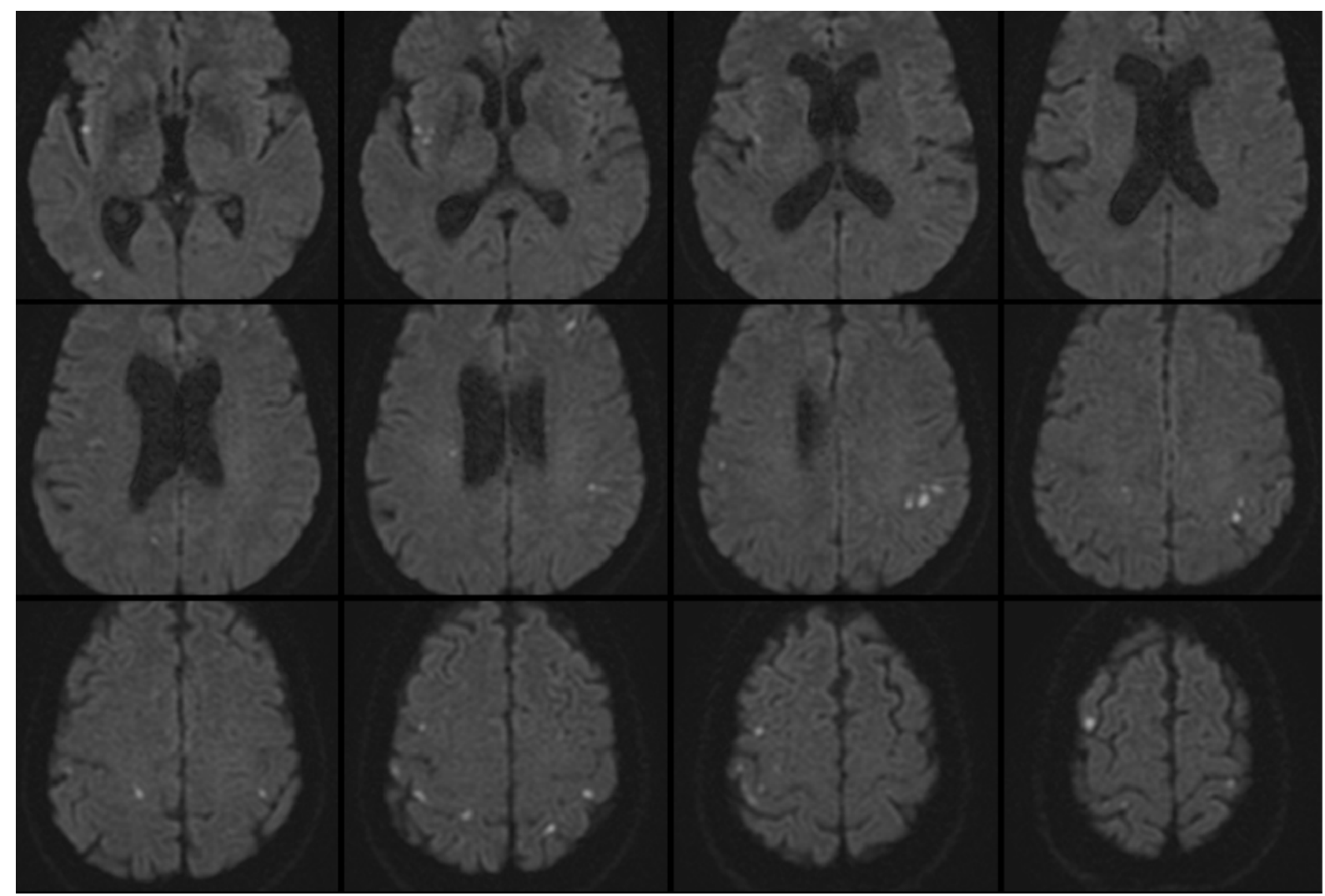

Figure 3 Multiple silent stroke embolization origin after thoracoscopic ablation for atrial fibrillation. MRI images (DWI, b=1,000) acquired 24 hours after ablation depict the occurrence small, multiple acute ischemic lesions situated mostly in the cortex; they were not detectable on pretreatment MRI scans. DWI, diffusion-weighted imaging; MRI, magnetic resonance imaging.

on early follow-up MRI, thus there was no reason for MRI follow-up in whole group of subjects.

\section{Discussion}

We evaluated the rate of silent strokes after 2 markedly different types of the invasive treatment for persistent or paroxysmal symptomatic AF: mini-invasive endovascular catheter and surgical thoracoscopic epicardial ablation. Our study shows a significantly higher rate of silent strokes after thoracoscopic epicardial ablation than after catheter ablation; $41 \%$ of patients were affected by silent strokes after the surgical procedure versus $7 \%$ of subjects after catheterization treatment. Moreover, clinicallymanifest stroke was diagnosed in one patient in the group treated surgically (see also Figure 4). The number of acute ischemic lesions after thoracoscopic epicardial ablation was surprisingly high, with a median of 6 new acute ischemic lesions per the affected subject. However, more than half
(54\%) of the lesions were reversible and no residual changes were detected on late follow-up MRI 6 months after therapy (see also Figure 7).

Both groups of treated patients were comparable according to the age, sex and most comorbidities. However, all surgically-treated patients had a history of nonparoxysmal AF versus $37 \%$ of patients in the catheter ablation group. In the group treated surgically, more patients suffered from chronic heart failure. This difference was due to the indication criteria for thoracoscopic ablation; non-paroxysmal AF was the main indication criterium, preferentially in combination with chronic heart failure. However, the preprocedural type of AF cannot explain the significantly higher number of lesions after the procedure, and no association was found by regression analysis.

We did not find any significant differences in pretreatment MRI findings; only $36 \%$ of subjects from the surgical group and $30 \%$ of subjects from the catheterization group had normal findings on brain MRI, the remainder 


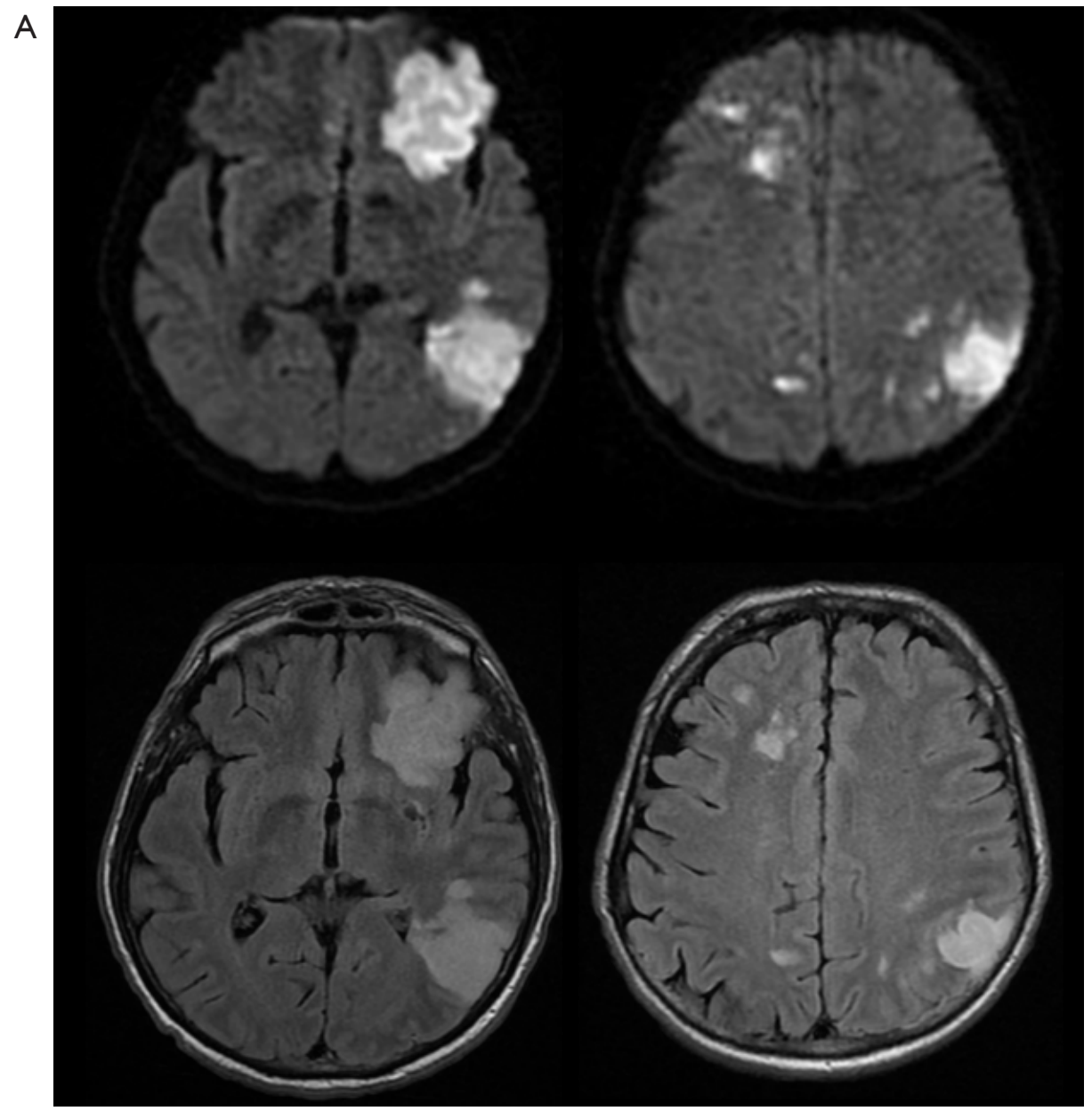

B

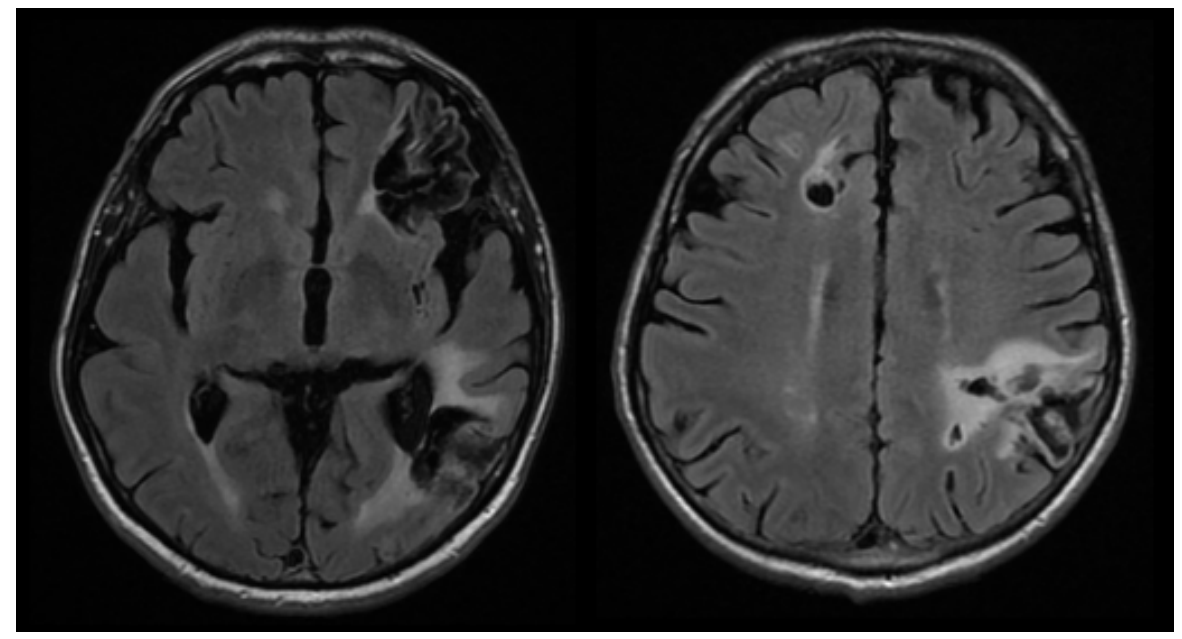

Figure 4 Manifest stroke embolization origin after thoracoscopic ablation for atrial fibrillation. Follow-up MRI demonstrates acute bilateral brain infarctions and their development in time. (A) MRI follow-up 24 hours after surgery. MRI images acquired 24 hours after ablation depict extensive territorial acute ischemic lesions in the left hemisphere and multiple smaller lesions in the right hemisphere. Upper row: DWI, b=1,000; bottom row: T2 FLAIR. (B) MRI follow-up 6 months after surgery. Chronic infarctions with pseudocystic transformation are shown on T2 FLAIR images. DWI, diffusion-weighted imaging; MRI, magnetic resonance imaging; T2 FLAIR, T2-weighted-fluidattenuated inversion recovery. 


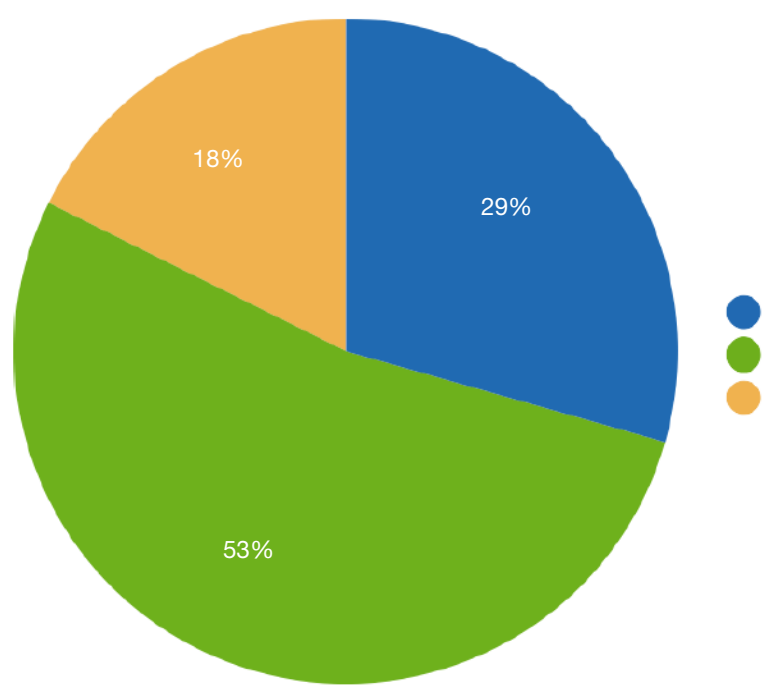

Complete reversible

Partial reversible

Transformation of lesions into chronic infarctions

Figure 5 Late follow-up MRI in patients treated with thoracoscopic epicardial ablation: patient-dependent development of lesions. The graph demonstrates that nearly in one-third of affected patients all acute ischemic lesions were completely reversible. MRI, magnetic resonance imaging.

Table 3 Acute ischemic lesions and their development after thoracoscopic epicardial ablation (17 patients affected)

\begin{tabular}{lccccc}
\hline Early follow-up MRI & $\mathrm{n}$ & \multicolumn{1}{c}{ Late follow-up MRI } & $\mathrm{n}$ & Percentage of reversible lesions \\
\hline No. of all acute ischemic lesions & 195 & No. of all residual chronic postischemic lesions & 89 & $53.5 \%$ \\
No. of acute lesions $<5 \mathrm{~mm}$ & 160 & No. of residual chronic postischemic lesions & 59 & $63.1 \%$ \\
No. of acute lesions sized $5-10 \mathrm{~mm}$ & 23 & No. of residual chronic postischemic lesions & 18 & $21.7 \%$ \\
No. of acute lesions sized 10-30 mm & 9 & No. of residual chronic postischemic lesions & 9 & $0 \%$ \\
$\begin{array}{l}\text { No. of acute lesions sized }>30 \mathrm{~mm} \text { and } \\
\text { territorial ischemia }\end{array}$ & 3 & No. of residual chronic postischemic lesions & 3 & $0 \%$
\end{tabular}

MRI, magnetic resonance imaging; No., number.

of subjects showed some signs of chronic vascular disease, which was more common in older patients. Moreover, isolated silent acute ischemic lesions were found in both groups of patients. These results are not surprising and are in an agreement with previously published findings. Disruption of normal atrial electromechanical function in $\mathrm{AF}$ leads to blood stasis and thrombus formation, most commonly in the left atrial appendage (5). Dislodgement or fragmentation of a clot can then lead to embolic phenomena; therefore, persistent $\mathrm{AF}$ poses a considerable risk for brain embolization $(5,12)$. It is well known that patients suffering from untreated AF have 3-5 times greater risk of manifest ischemic stroke (12); $18-25 \%$ of all ischemic strokes are associated with AF (2). Silent strokes occur twice as often in AF patients, even on anticoagulation therapy (13).
Gaita et al. found chronic, small ischemic lesions in approximately $90 \%$ of $\mathrm{AF}$ patients, the majority of them were on anticoagulation therapy, and patients that suffered from non-paroxysmal AF patients had a higher number of chronic ischemic lesions compared to paroxysmal patients (14). Accordingly, in the Reykjavik study, AF patients had significantly lower total brain volume compared with those without AF (15). As is generally accepted, the findings of chronic vascular changes and silent strokes on pretreatment MRI were likely caused by previous embolic events. We must state however, that not all silent strokes are linked to AF. Silent strokes from other etiologies are generally not rare events, especially in the elderly population $(16,17)$. This has been shown by progress in imaging methods, especially in MRI advancements with DWI (4). However, 
the prevalence of silent strokes from different sources in a population is not clear. According to a systematic review and meta-analysis by Fanning et al., the prevalence of silent strokes ranges from $5 \%$ to $62 \%$, with most studies in the $10 \%$ to $20 \%$ range (17). Longitudinal studies suggest an annual incidence of silent strokes between $2 \%$ and $4 \%$.

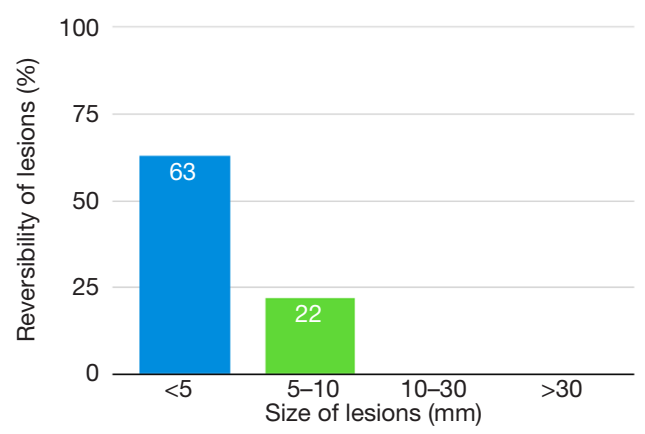

Figure 6 Reversibility of acute ischemic lesions according to diameter. The graph demonstrates reversibility of small lesions, in contrast to larger ones which typically transformed into chronic infarction.
There is a strong association between silent strokes and age, hypertension, carotid stenosis, chronic kidney disease and metabolic syndrome. Some studies have already reported an association of silent strokes with $\mathrm{AF}$, heart failure, coronary artery disease and even with advanced interatrial block $(17,18)$.

In our study, thoracoscopic epicardial ablation was associated with a significantly higher rate of silent strokes ( $41 \%$ of all treated subjects) in comparison to catheter ablation $(7 \%, \mathrm{P}<0.001)$. Moreover, most subjects suffered from multiple acute ischemic lesions, 194 lesions in 17 affected patients were found (as compared to only 3 lesions in 2 patients after catheter ablation). Most of the lesions $(82 \%)$ were smaller than $5 \mathrm{~mm}$ in diameter. Except for silent strokes, one patient $(2.6 \%)$ in the surgical group suffered from neurological deficit.

Each invasive cardiac procedure can lead to microembolisms and manifest or silent strokes. For instance, as recently published, transcatheter aortic valve implantation has been associated with a $2 \%$ risk of periprocedural manifest stroke, and asymptomatic brain micro-embolisms have been detected by MRI a few days after the procedure in approximately $80 \%$ of patients (9). For comparison we
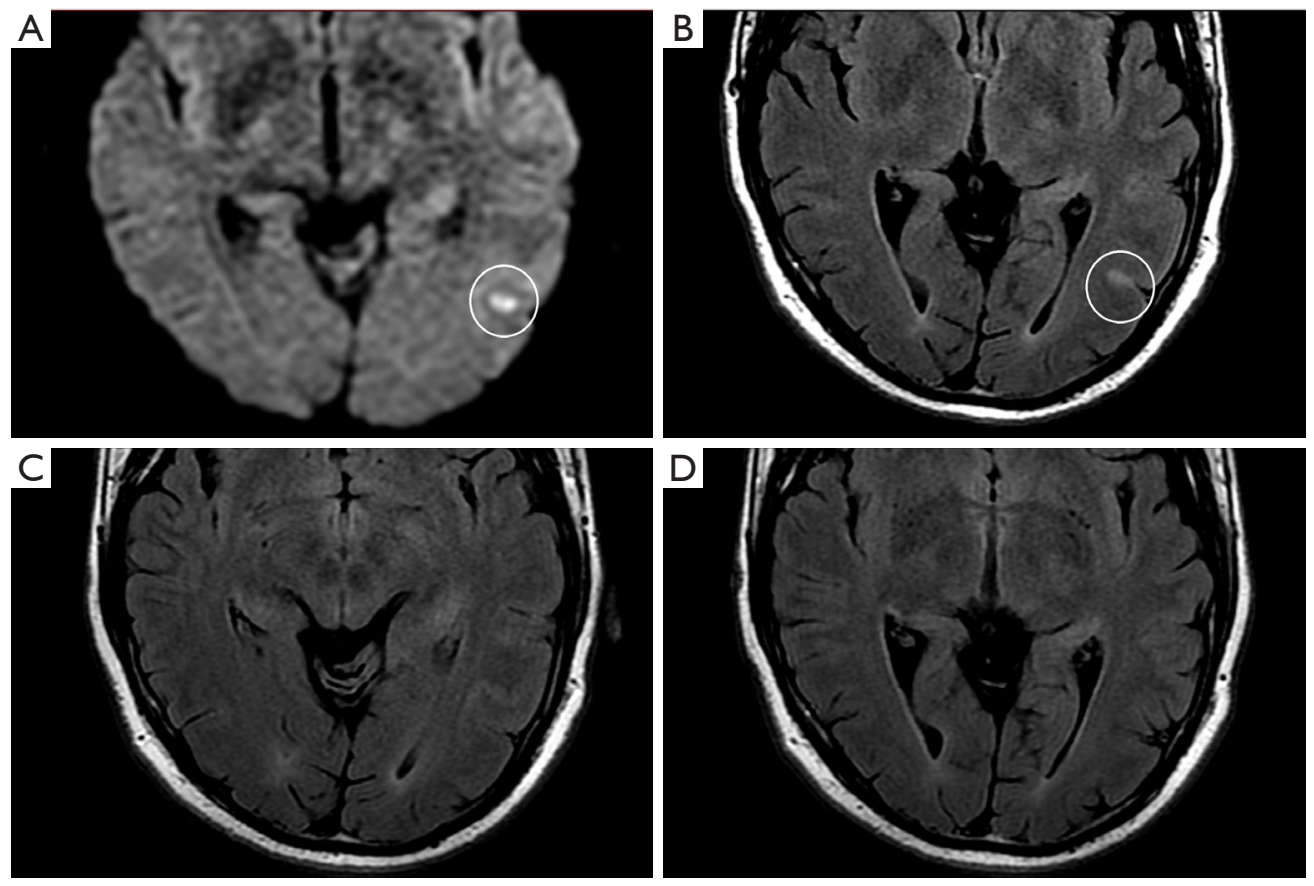

Figure 7 Reversible acute ischemic lesion. The early posttreatment MRI images acquired 24 hours after ablation depict a small acute ischemic lesion in the temporal lobe (circle) on both DWI; $b=1,000$ (A) and T2 FLAIR (B). This lesion was not detectable on T2 FLAIR MRI images acquired 6 months after ablation (C,D). DWI, diffusion-weighted imaging; MRI, magnetic resonance imaging; T2 FLAIR, T2weighted-fluid-attenuated inversion recovery. 


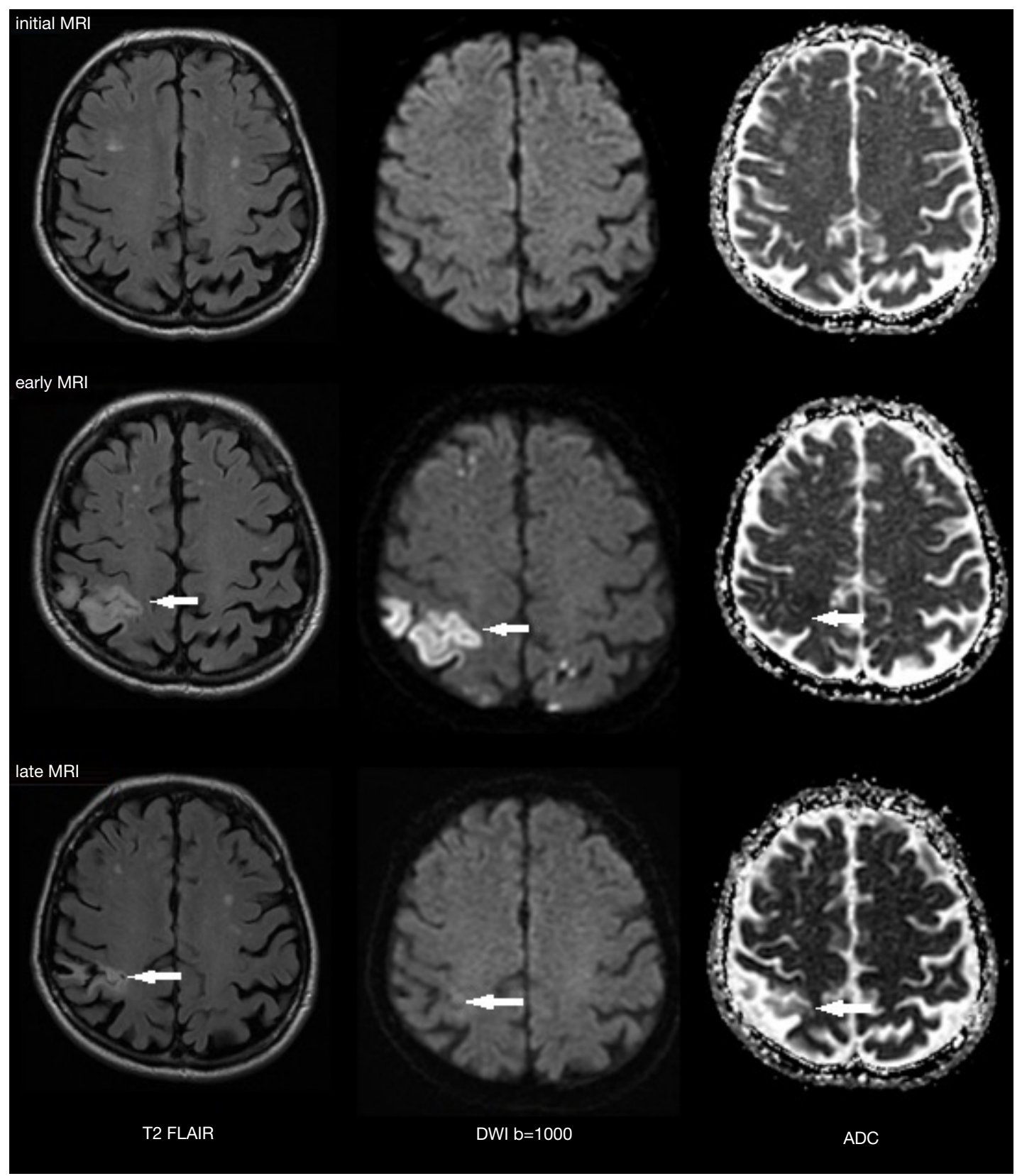

Figure 8 Transformation of acute ischemic lesions in time (irreversible and reversible lesions). Pretreatment MRI images (upper row) depict two small chronic lesions in the white matter. MRI images acquired 24 hours after surgery (middle row) depict substantial acute ischemic lesions in the right hemisphere (arrow) and several smaller lesions in both hemispheres. MRI examination 6 months after ablation (lower row), demonstrates transformation of the large acute ischemic lesion into chronic infarction (arrow) and also demonstrates the reversibility of some small lesions. ADC, apparent diffusion coefficient; DWI, diffusion-weighted imaging; MRI, magnetic resonance imaging; T2 FLAIR, T2-weighted-fluid-attenuated inversion recovery. 
also mention published data about silent strokes occurrence after open surgical aortic valve replacements; multiple silent strokes after that type of surgery are referred in $54 \%$ of subjects (19). After catheter ablation for AF, the periprocedural risk of manifest ischemic stroke ranges from $0.1 \%$ to $0.8 \%$ and there is a similar risk for transient ischemic attacks (20)$)$. However, manifest stroke seems to be only the tip of the iceberg, and the reported rate of silent strokes after catheter ablation is considerably higher and is reported over a wide range from $4 \%$ to $38 \%$, depending on the type of the catheter used, the anticoagulation protocol, patient characteristics, and MRI protocol (21). Most lesions reported after catheter procedures are small and solitary (21). In our study, we found only 3 lesions smaller than $5 \mathrm{~mm}$ in diameter in 2 patients, which we consider satisfactory.

The rate of silent strokes in our surgically-treated group was high and also one manifest stroke occurred. Several circumstances could have contributed to this fact. First, bridging from oral anticoagulation before the procedure and reduced anticoagulation before and immediately after the procedure could definitively increase the thrombotic risk during the procedure. However, in thoracoscopic ablation, the use of anticoagulation has to be balanced against the risk of bleeding. Moreover, vast majority of lesions achieved using epicardial thoracoscopic ablation as transmural, and thrombi could be formed on the endocardial scar tissue which is not irrigated and likely serves as a source for thrombi generation. Furthermore, atrial stunning is present after cardioversion, which has to be done in the majority of patients during or after thoracoscopic ablation, contrary to out-patient cardioversion, only reduced anticoagulation could have been used. Finally, the burden of general anesthesia could also contribute to the increased prothrombotic milieu.

On late follow-up MRI 6 months after thoracoscopic epicardial ablation, $53 \%$ of all acute ischemic lesions were reversible; we did not find any residual changes on late follow-up MRI. In 29\% of affected patients, all acute lesions completely disappeared. Very small lesions $<5 \mathrm{~mm}$ $(63 \%)$ were most commonly reversible. On the contrary, lesions greater that $10 \mathrm{~mm}$ in diameter were permanent and residual changes such as gliosis or pseudocystic transformation were always present on the late follow-up MRI (see also Figures 4 and 8). There are several possible explanations for these findings. In the case of reversible lesions, we likely detected reversible cytotoxic oedema and not the irreversible necrotic infarction core on DWI (22). It has been shown previously by angiographic studies that embolic obstruction can recanalize spontaneously, resulting in open arteries resupplying the ischemic brain area (23). All patients received anticoagulation peri-procedurally, thus small emboli in the supplying arterioles may have also been dissolved pharmacologically. Moreover, Koch et al. showed that the size of acute ischemic lesions measured on DWI overestimates the final size of definitive infarction (24). Most acute lesions that we detected were very small $(<5$ $\mathrm{mm}$ ), thus the residual lesion may not be detectable on scans with 4 or $4.5 \mathrm{~mm}$ slice thickness due to partial voluming and used slice gap in 2D sequences.

Our study has several limitations. First, although our study had a prospective design, it was a single center and non-randomized study, and the number of patients is limited for sensitivity analyses. Moreover, for thoracoscopic ablation, patients with non-paroxysmal AF were recruited, and preferentially with AF-induced heart failure. Second, the MRI protocols differed; the postoperative MRI protocol was abbreviated. Three-dimensional FLAIR or T2 sequences with better spatial resolution were not used; we used standard TSE T2 and T2 FLAIR sequences with a slice thickness of 4-4.5 mm. Therefore, some smaller lesions might not have been detected, especially on late followup MRI. Another consequence of using 2D sequences is the impossibility of volumetric measurement of the lesions, which would be more precise. Third, patients treated by catheter ablation were not scheduled for late follow-up MRI 6 months after therapy as we did not anticipate any diagnostically beneficial information would be gained in this group of patients.

We consider our study concerning the rate of silent strokes after different interventional procedures for $\mathrm{AF}$ as important and clinically relevant, as silent strokes are not necessarily without clinical significance. Silent strokes may be associated with subtle neurological deficits, cognitive dysfunction, as well as psychiatric disorders, and further with clinically-apparent stroke and early mortality $(14,15)$.

\section{Conclusions}

When comparing two different invasive procedures of $\mathrm{AF}$ treatment, periprocedural silent strokes were much more common after surgical thoracoscopic epicardial ablation than after catheter ablation. The majority of acute ischemic brain lesions were small, up to $5 \mathrm{~mm}$ in diameter, roughly half of which were reversible. The reversibility of acute brain lesions decreased with size; all lesions greater than 10 $\mathrm{mm}$ in diameter were persistent at follow up. However, in 
$29.4 \%$ of affected patients, all lesions were fully reversible.

\section{Acknowledgments}

Funding: This study was supported by the Czech Health Research Council grant AZV 16-32478A and by the Charles University research program PROGRES Q37.

\section{Footnote}

Conflicts of Interest: All authors have completed the ICMJE uniform disclosure form (available at http://dx.doi. org/10.21037/qims-21-35). Dr. HM serves as an unpaid Editorial Board Member of Quantitative Imaging in Medicine and Surgery. The other authors have no conflicts of interest to declare.

Ethical Statement: The study was approved by the Ethics committee of Faculty Hospital Kralovske Vinohrady, Prague, Czech Republic (EK-VP/28/3/2015). Written, informed consent provided by all patients.

Open Access Statement: This is an Open Access article distributed in accordance with the Creative Commons Attribution-NonCommercial-NoDerivs 4.0 International License (CC BY-NC-ND 4.0), which permits the noncommercial replication and distribution of the article with the strict proviso that no changes or edits are made and the original work is properly cited (including links to both the formal publication through the relevant DOI and the license). See: https://creativecommons.org/licenses/by-nc-nd/4.0/.

\section{References}

1. O'Donnell MJ, Xavier D, Liu L, Zhang H, Chin SL, RaoMelacini P, Rangarajan S, Islam S, Pais P, McQueen MJ, Mondo C, Damasceno A, Lopez-Jaramillo P, Hankey GJ, Dans AL, Yusoff K, Truelsen T, Diener HC, Sacco RL, Ryglewicz D, Czlonkowska A, Weimar C, Wang X, Yusuf S; INTERSTROKE investigators. Risk factors for ischaemic and intracerebral haemorrhagic stroke in 22 countries (the INTERSTROKE study): a case-control study. Lancet 2010;376:112-23.

2. Radu RA, Terecoasa EO, Bajenaru OA, Tiu C. Etiologic classification of ischemic stroke: Where do we stand? Clin Neurol Neurosurg 2017;159:93-106.

3. Hart RG, Diener HC, Coutts SB, Easton JD, Granger CB, O'Donnell MJ, Sacco RL, Connolly SJ; Cryptogenic
Stroke/ESUS International Working Group. Embolic strokes of undetermined source: the case for a new clinical construct. Lancet Neurol 2014;13:429-38.

4. Sacco RL, Kasner SE, Broderick JP, Caplan LR, Connors JJ, Culebras A, Elkind MS, George MG, Hamdan AD, Higashida RT, Hoh BL, Janis LS, Kase CS, Kleindorfer DO, Lee JM, Moseley ME, Peterson ED, Turan TN, Valderrama AL, Vinters HV; American Heart Association Stroke Council, Council on Cardiovascular Surgery and Anesthesia; Council on Cardiovascular Radiology and Intervention; Council on Cardiovascular and Stroke Nursing; Council on Epidemiology and Prevention; Council on Peripheral Vascular Disease; Council on Nutrition, Physical Activity and Metabolism. An updated definition of stroke for the 21st century: a statement for healthcare professionals from the American Heart Association/American Stroke Association. Stroke 2013;44:2064-89.

5. Wolf PA, Abbott RD, Kannel WB. Atrial fibrillation as an independent risk factor for stroke: the Framingham Study. Stroke 1991;22:983-8.

6. Donkor ES. Stroke in the 21st Century: A Snapshot of the Burden, Epidemiology, and Quality of Life. Stroke Res Treat 2018;2018:3238165.

7. Asad ZUA, Yousif A, Khan MS, Al-Khatib SM, Stavrakis S. Catheter Ablation Versus Medical Therapy for Atrial Fibrillation. Circulation: Arrhythmia and Electrophysiology 2019;12:e007414.

8. Budera P, Osmancik P, Herman D, Talavera D, Petr R, Straka Z. Risk of Intraatrial Thrombi After Thoracoscopic Ablation in Absence of Heparin and Appendage Closure. Ann Thorac Surg 2017;104:790-6.

9. Indja B, Woldendorp K, Vallely MP, Grieve SM. Silent Brain Infarcts Following Cardiac Procedures: A Systematic Review and Meta-Analysis. J Am Heart Assoc 2019;8:e10920.

10. Fazekas F, Kleinert R, Offenbacher H, Payer F, Schmidt R, Kleinert G, Radner H, Lechner H. The morphologic correlate of incidental punctate white matter hyperintensities on MR images. AJNR Am J Neuroradiol 1991;12:915-21.

11. The R Project for Statistical Computing. Available online: https://www.r-project.org/index.html

12. Bordignon S, Chiara Corti M, Bilato C. Atrial Fibrillation Associated with Heart Failure, Stroke and Mortality. J Atr Fibrillation 2012;5:467.

13. de Leeuw FE, de Groot JC, Achten E, Oudkerk M, Ramos LM, Heijboer R, Hofman A, Jolles J, van Gijn J, Breteler 
MM. Prevalence of cerebral white matter lesions in elderly people: a population based magnetic resonance imaging study. The Rotterdam Scan Study. J Neurol Neurosurg Psychiatry 2001;70:9-14.

14. Gaita F, Corsinovi L, Anselmino M, Raimondo C, Pianelli M, Toso E, Bergamasco L, Boffano C, Valentini MC, Cesarani F, Scaglione M. Prevalence of silent cerebral ischemia in paroxysmal and persistent atrial fibrillation and correlation with cognitive function. J Am Coll Cardiol 2013;62:1990-7.

15. Stefansdottir H, Arnar DO, Aspelund T, Sigurdsson S, Jonsdottir MK, Hjaltason H, Launer LJ, Gudnason V. Atrial fibrillation is associated with reduced brain volume and cognitive function independent of cerebral infarcts. Stroke 2013;44:1020-5.

16. Das RR, Seshadri S, Beiser AS, Kelly-Hayes M, Au R, Himali JJ, Kase CS, Benjamin EJ, Polak JF, O'Donnell CJ, Yoshita M, D'Agostino RB Sr, DeCarli C, Wolf PA. Prevalence and correlates of silent cerebral infarcts in the Framingham offspring study. Stroke 2008;39:2929-35.

17. Fanning JP, Wong AA, Fraser JF. The epidemiology of silent brain infarction: a systematic review of populationbased cohorts. BMC Med 2014;12:119.

18. Wang Z, Qin H, Chen G, Mok VCT, Dai Y, Cai Y, Cheng X, Qian Y, Chu M, Lu X. Association between advanced interatrial block and small vessel diseases in the brain. Quant Imaging Med Surg 2020;10:585-91.

19. Messé SR, Acker MA, Kasner SE, Fanning M, Giovannetti T, Ratcliffe SJ, Bilello M, Szeto WY, Bavaria JE, Hargrove WC 3rd, Mohler ER 3rd, Floyd TF. Determining Neurologic Outcomes from Valve Operations (DeNOVO) Investigators. Stroke after aortic valve surgery: results from a prospective cohort. Circulation 2014;129: 2253-61.

20. Haeusler KG, Kirchhof P, Endres M. Left atrial catheter ablation and ischemic stroke. Stroke 2012;43:265-70.

21. Deneke T, Jais P, Scaglione M, Schmitt R, DI Biase L, Christopoulos G, Schade A, Mügge A, Bansmann M, Nentwich K, Müller P, Krug J, Roos M, Halbfass P, Natale A, Gaita F, Haines D. Silent cerebral events/lesions related to atrial fibrillation ablation: a clinical review. J Cardiovasc Electrophysiol 2015;26:455-63.

22. Copen WA. Multimodal imaging in acute ischemic stroke. Curr Treat Options Cardiovasc Med 2015;17:368.

23. Dalal PM, Shah PM, Sheth SC, Deshpandi CK. Cerebral embolism: Angiographic observations on spontaneous clot lysis. Lancet 1965;1:61-4.

24. Koch S, McClendon MS, Bhatia R. Imaging evolution of acute lacunar infarction: leukoariosis or lacune? Neurology 2011;77:1091-5.
Cite this article as: Malikova $\mathrm{H}$, Kremenova $\mathrm{K}$, Budera $\mathrm{P}$, Herman D, Weichet J, Lukavsky J, Osmancik P. Silent strokes after thoracoscopic epicardial ablation and catheter ablation for atrial fibrillation: not all lesions are permanent on followup magnetic resonance imaging. Quant Imaging Med Surg 2021;11(7):3219-3233. doi: 10.21037/qims-21-35 